\title{
Sistemas de innovación y formación de redes para el desarrollo local
}

\author{
Innovation Systems and Networks Formation for local development
}

\author{
Carlos Alberto Hernández Medina ${ }^{1}$ \\ Alexander Báez Hernández ${ }^{2}$ \\ Magdalys Alibet Carrasco Fuentes ${ }^{3}$
}

\section{Resumen}

Se abordó el concepto de red local de gestión del conocimiento como base de un Sistema de Innovación Local para Cuba. Se aplicó una metodología hermenéutica a un nivel interpretativo. La red se vio como una forma de incorporar vínculos e interacciones que hacen posible, en lo local, regional y nacional, la existencia de economías externas y rendimientos crecientes. El reconocimiento cada vez mayor obtenido por las redes se sustentó en que, independientemente de la cantidad de información y conocimiento codificado que produzca la sociedad, cuando no hay vinculación entre el conocimiento codificado y las competencias encarnadas en los individuos y organizaciones, no es posible captar el impulso productivo del conocimiento, plasmarlo en innovación y en un sendero de desarrollo sostenible.

Palabras clave: Conocimiento tácito; desarrollo local; gestión del conocimiento; redes.

\section{Summary}

The concept of the local knowledge management network was addressed as the basis of a Local Innovation System for Cuba. Hermeneutical methodology was applied at an interpretive level. The network was seen as a way of incorporating links and interactions that make possible, locally, regionally and nationally, the existence of external economies and increasing returns. The increasing recognition obtained by networks was based on the fact that, regardless of the amount of information and coded knowledge that society produces, when there is no link between coded knowledge and the skills embodied in individuals and organizations, it is not possible to capture the productive impulse of knowledge, translate it into innovation and a path of sustainable development.

1 Master en Agricultura Sostenible, Coordinador de Investigaciones, Centro Universitario Municipal (CUM) Camajuaní. Independencia \# 126-B. Camajuaní 5250o. Villa Clara, Cuba. cahm862@uclv.edu.cu http://orcid.org/oooo-ooo3-3446-3014

2 Master en Contabilidad Gerencial, Profesor de la Facultad de Ciencias Administrativas. Universidad Central de Ecuador, Avenida de las Américas, Quito, Ecuador. abaez@uce.edu.ec

3 Master en Ciencias de la Educación, Coordinadora de la carrera de Sociología, Centro Universitario Municipal Camajuaní. Joaquín Paneca \# 62-A. Camajuaní. Villa Clara, Cuba. alibet7305@uclv.edu.cu

Recibido: 05/11/2019 Aprobado: 20/05/2020 
Keywords: Tacit knowledge; local development; knowledge management; networks

\section{Introducción}

En los países desarrollados se dedican grandes recursos para asegurar la apropiación y uso intensivo del conocimiento para acceder a mercados donde el valor agregado a los productos determina la supervivencia competitiva. Su objetivo es captar conocimiento relevante y capitalizarlo en sus productos, asegurando crear riqueza para grandes empresas monopolistas. Poseer conocimiento expresado en el saber cómo es determinante y el dinero pagado se asume como inversión estratégica que se realiza y justifica en la capacidad del conocimiento relevante para generar valor agregado. Así surgió la gestión del conocimiento, tema emergente de interés académico y profesional contemporáneo (Hernández, et al., 2017).

En la bibliografía predomina una orientación economicista sobre la gestión del conocimiento. Esa es una regularidad internacional porque la producción teórica sobre gestión del conocimiento procede de los países industrializados. En su mayoría está rigurosamente encaminada hacia la economía en detrimento de lo social. En América Latina la realidad no difiere mucho, aunque existen ejemplos muy interesantes en Argentina, Venezuela y Brasil. Esa regularidad impone para su aplicación en Cuba, la necesidad de despojar a la gestión del conocimiento de la esencia del sistema de dominación donde se creó y adecuarla a las condiciones del país (Hernández et al., 2013).

En Cuba, el Programa Ramal Gestión del Conocimiento para la Innovación y el Desarrollo (GUCID), fue un esfuerzo comprometido del Ministerio de Educación Superior para contribuir al desarrollo local. Surgió en 2006 por iniciativa de la Cátedra CTS+I de la Universidad de La Habana, abanderada de los estudios de desarrollo local en el país. De aquel Programa Ramal surgió la Red GUClD donde participan con sus proyectos gran parte de las universidades cubanas, Centros de Investigación y Centros Universitarios Municipales de varias provincias. Entre ellos se destacan los Centros Universitarios Municipales (CUM) Yaguajay, Remedios, Camajuaní, Placetas, Mayarí, El Salvador, La Palma y Jatibonico.

A partir de objetivos iniciales que posibilitan dirigir las acciones, cada actor involucrado en GUCID estudia y documenta su realidad local y adquiere conocimiento relevante sobre sus potencialidades para el desarrollo local. La idea inicial de esta investigación, que se inició en 2007, surgió a partir de dos proyectos iniciales en ese Programa (MES, 2012a y MES, 2012b).

La municipalización de la Educación Superior que dio lugar a la creación de los Centros Universitarios Municipales (CUMo y el proceso de transformar el modelo socioeconómico cubano crearon oportunidades no aprovechadas con eficacia aún en 


\section{CIENCIAS SOCIALES}

contextos locales por resistencia de la práctica tradicional que obstaculiza desplegar la gestión del conocimiento para catalizar procesos de innovación (Schulze, 2003).

La capacidad del municipio para desarrollarse depende en lo fundamental de la disposición de los actores locales expresada en niveles de cohesión social y en cómo aplican productivamente el conocimiento que poseen o captan. Sin embargo, esos saberes diversificados pueden coexistir junto a los problemas sin lograr soluciones. Esos vínculos necesitan una concepción comunitaria para identificar, adquirir, desarrollar, difundir, utilizar y retener conocimiento presente en la vida social local o fuera de ella. (Garcés, 2013).

Eso es posible si se fortalece una participación en la que mecanismos de deliberación eficaces y responsabilidad de los actores son elementos constitutivos de la capacidad de la sociedad local para ejecutar una política de desarrollo viable y consensuada. (Cravacuore, 2010).

El presente estadio de desarrollo se caracteriza por el paso gradual de un sistema productivo basado en la estandarización, a uno focalizado en la variedad y calidad de productos y procesos productivos. Nuevos factores reemplazan la tierra, el trabajo y el capital físico. Recursos naturales, ambientales, humanos y tecnología se convierten en elementos clave debido a la Revolución Tecnológica (Hernández et al., 2017).

Este artículo pretende hacer una revisión del estado del arte en cuanto a la formación de redes como proceso que fortalece la creación de Sistemas de Innovación Local en función de lograr el desarrollo local en Cuba.

\section{Revisión de literatura}

Es clara la importancia de las relaciones que se establecen entre actores y agentes que componen un sistema económico-social como fuente para su desarrollo como sistema. Los vínculos entre empresas y sectores cobran especial relevancia en un sistema más integrado, donde la internacionalización de la producción y el comercio es cada vez más importante. Si los países quieren insertarse en senderos de crecimiento sostenido es fundamental que reconozcan en dichos vínculos un factor clave para lograrlo (Cimoli, 2005).

El surgimiento de un sistema de redes como expresión de disposiciones institucionales e industriales para la difusión de los vínculos sistémicos entre actores locales es condición necesaria para avanzar por el sendero del desarrollo local sostenible. Por eso las redes son una categoría que adquiere cada vez mayor importancia para saber si un país, región o localidad es capaz de generar un sendero alternativo hacia el desarrollo sustentable (Hernández et al., 2015). 
Tanto las estrategias de desarrollo, como las políticas de equilibrio territorial predominantes durante décadas en América Latina, se han sustentado en el paradigma del desarrollo polarizado, propio del industrialismo sustitutivo, donde la organización productiva responde a un esquema institucional centralista, regido por un crecimiento nacional planificado desde el gobierno nacional.

Para ese enfoque tradicional, el desarrollo es posible gracias a la construcción de infraestructuras y la continua expansión de empresas industriales localizadas preferentemente en grandes centros urbanos. La tipología de la innovación tecnológica introducida y las políticas económicas, demandan la consecución de economías de escala y externas. Así se diseñaron las políticas tradicionales en Cuba, siguiendo una tendencia general respecto a entender el desarrollo promovido desde arriba (Báez et al., 2018).

Desde la década del 90 se genera una situación de crisis y reemplazo de viejos paradigmas, que pasa de la visión redistributiva al potencial de desarrollo en el territorio y provoca un cambio significativo en la conceptualización y aplicación de la política de desarrollo regional. La visión del desarrollo endógeno introduce como estrategia dominante el desarrollo difuso, desde abajo, en que el espacio asume la connotación de factor estratégico para el desarrollo del territorio (Báez et al., 2018a). El foco de análisis se ha trasladado desde la problemática de la redistribución a crear recursos e incorporar mecanismos de desarrollo.

Esto hace necesario construir entornos innovadores en cada territorio y marca la insuficiencia del enfoque tradicional del desarrollo, basado en factores productivos. Según Alburquerque (1998), las ventajas competitivas dinámicas no se basan tanto en la dotación de recursos naturales o salarios bajos, como en hacer innovación tecnológica, organizativa y estratégica; en usar el conocimiento.

\section{Metodología}

Para el logro del objetivo planteado en la investigación se utilizaron diversos métodos. Se partió de la hermenéutica en la revisión de documentos, artículos, informes de investigación y trabajos presentados en eventos científicos que pudieran ofrecer información sobre la formación de redes, los sistemas de innovación, proyectos de desarrollo local y estudio de casos sobre estos temas.

Del nivel teórico usamos: el método analítico y el sintético, el lógico y el histórico y el inductivo-deductivo. Las técnicas utilizadas en la investigación son: la revisión de documentos facilitados por las instituciones comprometidas con el proceso de desarrollo local y la vida económica y productiva de los territorios como: Asamblea Municipal del Poder Popular, Dirección Municipal de Economía y Planificación, Delegación Municipal de la Agricultura, Asociación Nacional de Agricultores Pequeños y Centro 
Universitario Municipal. También se utilizó la entrevista en algunos casos para corroborar y actualizar la información recogida a partir de los documentos estudiados.

\section{Resultados y discusión}

La toma de decisiones en función del desarrollo en el ámbito local, regional y nacional no está determinada exclusivamente por la presión en la demanda de productos o servicios. Responde también a factores especiales que provienen de la organización de los procesos productivos y sus rutinas dominantes. En este mundo globalizado los distintos vínculos e interacciones entre ellos han adquirido nuevo carácter y mayor importancia (Báez et al., 2018).

Para los países subdesarrollados del Sur, exportadores de materia prima, son una estructura capaz de generar un sendero alternativo hacia el desarrollo. Según Hirschman (1977) algunas de las principales características del desarrollo de un país en el período de crecimiento inducido por las exportaciones se pueden describir en términos de los vínculos que surgen de sus productos principales.

Un elemento básico a tener en cuenta a la hora de analizar los sistemas de innovación es que los nexos e interacciones sistemáticos no sólo se refieren a las empresas sino también, en general, a los encadenamientos entre empresas, organizaciones y marcos institucionales que cada sociedad genera como forma propia de organización social. Así, el concepto de redes puede considerarse como variable de observación para comprender si los encadenamientos e interacciones sistemáticos se difunden a los sistemas innovadores (Carrasco et al., 2015).

Las redes pueden verse como una forma de incorporar vínculos e interacciones que hacen posible, en el nivel regional y local, la existencia de economías externas y rendimientos crecientes. En tal sentido, el reconocimiento cada vez mayor que tienen las redes está sustentado en la idea de que independientemente de la cantidad de información y conocimiento codificado que produzca una sociedad, si no hay vinculación entre la cantidad total de conocimiento codificado y las diversas competencias encarnados en los individuos y las organizaciones, el país no será capaz de captar el impulso productivo del conocimiento y plasmarlo en innovación y en un sendero de desarrollo estable (Soete, 1996; Dosi, 1998; Cimoli y De la Mothe, 2001).

Las empresas son los actores principales de las redes porque están integradas a través de un gran complejo de vínculos con otras empresas e instituciones. La forma en que una empresa se integra a una red determina su capacidad para captar economías externas e incrementar los rendimientos. A nivel local eso pone de relieve el papel central de las interacciones entre empresas integradas en redes, en su rivalidad o pugna por el mercado, como el factor de mayor peso en la configuración de un sistema de innovación capitalista y su cambio estructural. En Cuba, Hernández et al. (2015a) 
incluyen en las redes a otros actores sociales como Universidades, gobierno, sociedad civil y organizaciones profesionales.

Los cambios estructurales de los sistemas de innovación de los países de América Latina han seguido un sendero que se vio afectado intensamente por crisis económicas generalizadas, intensidad y ritmo de la neo-liberalización y cambios en los precios internacionales que afectaron radicalmente la estructura de los mercados y el papel desempeñado por las empresas. Ese proceso contribuye a desarrollar aún más las redes de conocimiento en los países de economía más desarrollada a costa de nuestras economías, aumentando la brecha tecnológica y de conocimiento entre Norte y Sur.

Coincidimos con Báez et al. (2018) en que, la internacionalización de la producción que se está produciendo actualmente en las economías de América Latina un factor que contribuye a incrementar la demanda de conocimientos y de actividades en red, dirigida hacia las economías más desarrolladas para perjuicio de los países más pobres.

El concepto de sistema de innovación no es suficiente en el nivel local para comprender las causas de la creciente brecha espacial y temporal que, existe entre la generación del conocimiento y su difusión y uso para la innovación tecnológica en nuestros países subdesarrollados. Creemos que esta brecha está dada fundamentalmente por la dificultad existente para generar políticas que acerquen los intereses de los que producen conocimiento y los que deben utilizarlo y optimizar el proceso de producción de bienes materiales y de servicios.

Los cambios estructurales de los sistemas de innovación de los países de América Latina han seguido un sendero que fue afectado intensamente por la globalización generalizada en lo económico. Una característica fundamental de la globalización es la intensidad y ritmo del proceso de liberalización y los incentivos introducidos por cambios en los precios internacionales que afectan radicalmente la estructura del mercado y el papel desempeñado por las empresas locales.

La innovación se concibe como proceso interactivo, no incumbe a una empresa aislada. Exige un proceso de búsqueda activa de nuevas fuentes de conocimiento y tecnología para aplicarlos a procesos de producción de bienes y servicios. Es un proceso social que se desenvuelve mejor cuando se logra mayor interacción entre proveedores y usuarios de bienes, servicios, conocimiento y tecnología. Incluye a las organizaciones e infraestructura pública como universidades y centros de investigación (Freeman, 1994; Nelson, 1998).

Según Misa (1991) a nivel local, una red es un concepto social que pretende englobar a [...] las organizaciones y personas comprometidas con el sistema por diversos intereses, a las corporaciones dedicadas a la fabricación, a los laboratorios de 


\section{CIENCIAS SOCIALES}

investigación y desarrollo, a los bancos de inversión, las instituciones educativas y a los organismos reguladores.

A su vez, estos vínculos al interior de las redes están sustentados por una inversión cada vez mayor y por el carácter local de la mayoría de las actividades de aprendizaje. Determinan las oportunidades y limitaciones a las que se enfrenta cada proceso de innovación y difusión de conocimiento (Hernández et al., 2017).

Las empresas buscan vínculos para fomentar el aprendizaje interactivo entre ellas y que las redes y socios externos aporten activos complementarios. Esto ayuda a repartir entre un número mayor de organizaciones los costos y riesgos derivados de la innovación para acceder a los resultados de nuevas investigaciones, a adquirir componentes tecnológicos clave para productos o procesos nuevos y a compartir activos en los procesos de fabricación, marketing y distribución (Báez et al., 2018).

Las redes son una categoría muy importante para comprender y explicar el funcionamiento de los sistemas de innovación. Coordinan y regulan el papel complementario que desempeñan la cooperación, coordinación y competencia al analizar los patrones de cambio y el mejoramiento del desempeño económico. Cuando están organizadas de manera conveniente, las redes son un poderoso motor que estimula el progreso y el desarrollo económico. Cuando su organización y conectividad son deficientes, pueden inhibir en gran medida el proceso de innovación (Carrasco et al., 2015).

La eficacia del sistema económico, en la asignación de recursos a ciertas funciones económicas y difusión de innovaciones técnicas y organizacionales que, depende cada vez más de la posibilidad de acceso al conjunto de nexos dentro de la red y del acceso a los flujos de conocimientos que se necesitan en un contexto institucional específico (Dosi et al., 1990).

Los autores han notado que la competencia y la eficiencia en nuestro mundo globalizado no están generadas por los que actúan en forma aislada, sino por redes constituidas por organizaciones disímiles, públicas y privadas. Estas se adaptan mejor a los rápidos cambios de las exigencias e innovan sus productos y procesos ya que el desarrollo tecnológico tiene un ritmo muy alto. Para reducir los riesgos y acortar el tiempo para lograr un producto nuevo o mejorado, se especializan y optimizan el proceso de innovación con mayor apoyo en el conocimiento y el saber cómo que aportan otros miembros de sus redes.

El auge de las Tecnologías de la Información y las Comunicaciones (TIC), constituye un ambiente nuevo caracterizado por la incertidumbre tecnológica; pero, implica nuevas oportunidades y desafíos para operar en redes. La aparición de nuevas tecnologías tiene consecuencias importantes para la economía de los países de América Latina (Hernández et al., 2017). 
El conocimiento tácito y local desempeña un papel fundamental en la mayoría de las actividades de innovación (Garcés, 2013). El proceso de innovación tiene dos aspectos en común con el conocimiento: uno es que es un elemento público en potencia, y el otro es que es un elemento tácito. El elemento público consiste en el conocimiento asequible que sólo en potencia está al alcance de quien lo procura, puesto que existen distintas formas de conceptualizarlo y de codificarlo.

El elemento tácito surge de un concepto desarrollado por Polanyi (1967), diciendo que estaba relacionado con [...] "aspectos del conocimiento y de la percepción intuitiva y con otras aptitudes propias de los individuos, que están mal definidas, no codificadas o no tienen estado público y que, por lo tanto, ni siquiera los propios individuos pueden expresar en su totalidad; aptitudes estas que difieren de una persona a otra, pero que pueden ser compartidas en gran medida entre colaboradores y colegas que llevan adelante una experiencia en común" [...]

El análisis anterior nos confirma que hay dos dimensiones de la Innovación, una de carácter industrial que implica una dimensión pública, y otra privada del conocimiento y la tecnología. Además, se puede hacer una distinción entre la tecnología y la información: mientras la información trasciende los límites de las empresas, la tecnología implica un conocimiento tácito y específico que no es ni puede ser registrado por escrito en proyectos y planos y que, por ende, no puede ser difundido exhaustivamente, ni como información pública ni como información patentada o de propiedad exclusiva.

El hecho de que dicho conocimiento tácito resida principalmente en los individuos, las organizaciones y las redes es de particular importancia. Donde las empresas que compiten entre sí están sólidamente integradas en una red formada por otras empresas, instituciones e infraestructuras, pueden capturar las externalidades y el conocimiento que se generan en la misma red. La apropiación de las externalidades y la difusión de conocimiento en un grupo restringido de empresas permitirán que las mismas muestren funciones de costo medio decreciente. Como consecuencia de lo anterior, las empresas compiten según su capacidad para capturar las interrelaciones tecnológicas, las economías de escala en la producción, y según su aptitud para incorporar el aprendizaje colectivo a través de la interacción y la distribución de conocimientos. Ese patrón de competencia define barreras de entrada a la red diferentes para empresas distintas, según la ventaja competitiva que cada una de las respectivas redes pueda desarrollar en términos de su función de costo decreciente y su escala de producción.

\section{Conclusiones}

1. Las redes son un mecanismo fundamental a través del cual se reducen los compromisos irreversibles y los costos hundidos, y generalmente brindan una flexibilidad mayor la cual se puede lograr a través de la internalización. 


\section{CIENCIAS SOCIALES}

2. El modelo de trabajo en red se concibe como un elemento importante para explicar cómo se configuran los Sistemas de Innovación Local en Cuba.

3. La competencia entre los actores que forman parte de una red es el principal factor que explica la configuración de la red y sus cambios a través del tiempo.

4. Las grandes desventajas de las empresas locales en su capacidad de crear conocimientos y difundir las innovaciones, pueden atenuarse en lo esencial en el marco de los mecanismos de auto-fortalecimiento generados en las redes.

5. En Cuba, las Redes de Gestión del Conocimiento y la Innovación para el desarrollo local cada vez involucran a mayor cantidad de actores sociales.

\section{Agradecimiento}

Esta publicación obtuvo el financiamiento de: El Fondo de Asistencia Internacional de los Estudiantes y Académicos Noruegos (SAIH).

\section{Lista de referencia}

Alburquerque, F. (1998). Cambio Tecnológico, Globalización y Desarrollo Económico Local. Instituto de Economía y Geografía, Consejo Superior de Investigaciones Científicas. Madrid.

Báez, A.; Hernández, C.; Carrasco, M. A. (2018). Praxis de una red local GUCID y desarrollo local. Rev. Ingeniería Industrial. Año 18. Nº 3 . pp. 253-266, 2018.

Báez, A.; Hernández, C.; Perdomo, J. M.; Garcés, R.; Carrasco; M. A. (2018a). Modelo de gestión del conocimiento para el desarrollo agropecuario local. Revista Estudios Sociales. Revista de Alimentación Contemporánea y Desarrollo regional. No. 51, Vol. 28. pág.: 2 - 26. Enero - Junio. 2018. ISSN: 2395-9169. DOI: https:// dx.doi.org/10.24836/es.v28i51.517

Carrasco, M., Hernández, C. Fernando, S., Vera-Cruz, A. (2015). Integration of university projects to local development: Case Study of Camajuaní municipality. Proceedings. GLOBELICs 2015. Habana. 23-25 sept.

Cimoli, M. (2005). Redes, estructuras de mercado y shocks económicos. CEPAL- GTZ. En: Cimoli, M. (2005). Cambios estructurales de los sistemas de innovación en América Latina. Redes Jerarquías y Dinámicas Productivas. Naciones Unidas, Santiago de Chile.

Cimoli M., De la Mothe J. (2001). The Governance of Technology and Development. En: De la Mothe J. (Ed.) Science,Technology and Governance. Pinter Publisher. London. 
Cravacuore, D. (2010). La articulación de actores para el desarrollo local. VII Congreso de la SAAP. http://www.saap.org.ar/esp/docscongresos/congresos-saap/VII/ programa/paneles/d/d1/cravacuore.pdf

Dosi G., L. Tyson, Zysman J. (1990). Trade, Technologies and Development: framework for discussing Japan. En Johnson C., Tyson, L., Zysman J. (Eds.) Politics and Productivity. Ballinger Publishing. New York.

Dosi G. (1999). Some Notes on National Systems of Innovation and Production, and their Implication for Economic Analysis. En Archibugi D, et al. (Eds.) Innovation Policies in a Global Economy. Cambridge Univ. Press. London.

Freeman C. (1994). Technological Revolution and Catching up: ITC and the NICs. En J. Fagerberg, N. Von Tunzelman, B. Verspagen (Eds.) The Dynamics of Technology, Trade and Growth. Edgar Elgar. London.

Garcés, R. (2013). La gestión del conocimiento en las condiciones del municipio de Remedios como contribución a su desarrollo local. Tesis Doctoral en Ciencias Sociológicas. Santa Clara: Centro de Estudios Comunitarios. Fac. C. Sociales. Universidad Central "Marta Abreu" de Las Villas.

Hernández, C.; Carrasco, M.; Garcés, R.; Casas, R. (2015). Vinculation of Municipal University Center (MUC) in innovation local network. Case study of Camajuaní, Cuba. Proceedings GLOBELICs 2015 Congress. Ciudad de La Habana. 23 -25 septiembre.

Hernández, C.; Carrasco, M.; Garcés, R. (2015a). Collaborations and links of Municipal University Centre Local Networks of Knowledge and Innovation: Case Study of Camajuaní, Cuba. Proceedings. GLOBELICs 2015. Habana, Cuba. 23-25 setiembre.

Hernández, C.; Garcés, R.; Perdomo, J. M.; Carrasco, M. A. (2017). Gestión universitaria del conocimiento para el desarrollo local. Revista Lámpsakos. No.18. pp. 44-57. julio-diciembre. 2017. ISSN: 2145-4086. DOI: http://dx.doi. org/10.21501/21454086.2317

Hirschman A. (1977). Generalized linkage approach to development, with special reference to staples. En Nash, M. (Ed.) Essays on Economic Development and Cultural Change in Honor of B. F. Hoselitz. University of Chicago Press.

MES (2012a). Informe del Grupo de Expertos No.1. Producción de Indicadores de desarrollo local. Taller Nacional "Formación y medición para la GUCID". La Habana: Programa Ramal GUCID. 
MES (2012b). Informe del Grupo de Expertos No. 2. Indicadores para la GUCID. Taller Nacional "Formación y medición para la GUCID". La Habana: Programa Ramal GUCID.

Misa, T.J. (1991). Constructive Technology Assessment: Cases. Concepts, Conceptualization. Conference on Constructive Technology Assessment. Twente. The Netherlands.

Nelson, R. (1998). The Agenda for Growth Theory: a Different Point of View. Cambridge Journal of Economics. No. 22: 497-520.

Polany, M. (1967). The Tacit Dimension. Doubleday Anchor. New York.

Schulze, A. (2003). Knowledge management in innovation processes. En: Zedtwitz., M. (2003). (Ed.) Management of Technology: Growth through Business Innovation and Entrepreneurship, Selected Papers from the Tenth International Conference on Management of Technology. (pp. 265-280). Netherlands: Ed. Pergamon.

Soete, L. (1996). The Challenges of Innovation. Seville. IPTS Report. Sept. 1996. 